\title{
From Micro-Patterns To Nano-Structures By Controllable Colloidal Aggregation At Air-Water Interface
}

\author{
Xiaorong Xiong, Kerwin Wang and Karl F. Böhringer \\ Department of Electrical Engineering, University of Washington \\ Seattle, WA 98195-2500, USA
}

\begin{abstract}
In this paper, we discuss a method to transform traditionallithography micro patterns into nano-structures by selfassembly of nano-beads. In our approach, the destined substrate is prepared with hydrophilic micro patterns on hydrophobic background. Colloid with nano-beads wets exclusively the hydrophilic patterns due to interfacial forces when passing through air-water interface. After evaporation of water from the colloid, three-dimensional nano-bead structures are formed. A geometric model is proposed to describe this self-assembly process and its dependence on bead size, concentration, and pattern geometry, which can provide control over the aggregation of three-dimensional nano-structures.
\end{abstract}

\section{INTRODUCTION}

With recent successful developments in functional nanostructures, e.g. nano-bead or carbon-nanotube sensors, their control and manipulation at the nano-scale becomes a challenging problem. A variety of self-assembly methods have been demonstrated by using biological, chemical, electrical or magnetic driving forces [1-4]. All these approaches require specific treatments or coatings of the nano-devices. For example, single stranded DNA has been attached to nano-beads to create the driving force from DNA strand binding [1]. In this paper, we discuss a method to selfassemble and arrange nano-beads by using surface tension.

At the micro-scale, surface tension driven fluidic selfassembly techniques have been demonstrated successfully with a wide variety of devices ranging from a hundred of microns to several millimeters [5-7]. Different from prior work at the micro-scale, we adapt this fluidic assembly technique into the nano-domain by taking advantage of colloid aggregation at the air-water interface [8,9]. By controlling the colloid volume and dilution, the aggregation of beads can be precisely arranged into nano-structures that are one to two orders of magnitude smaller than the original pattern. In addition, this technique can be applied to general nano-devices without specific surface treatment. Presented are the principles, models, methods and results of this approach.

\section{Principle AND Models}

Fig. 1 illustrates the self-assembly process. The substrate is prepared with hydrophilic patterns on hydrophobic background (Fig. 1a). When the substrate covered with the colloid passes through the air-water interface, the excessive colloid is removed from the substrate and the colloid wets exclusively the hydrophilic areas (Fig. 1b). As a result of surface tension force and dragging effect from the receding air-water interface due to evaporation (Fig. 1c), the nanobeads are arranged on the surface in predictable patterns (Fig. 1d).

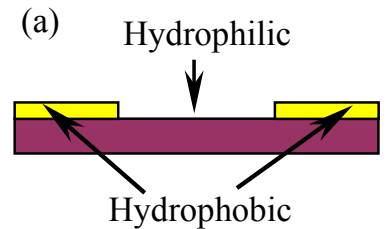

(c)

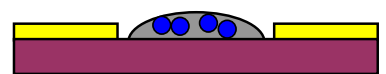

(b)

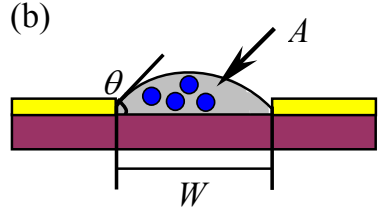

(d)
Figure 1. (a) A substrate prepared with hydrophilic binding sites on hydrophobic background. (b) When the colloid is applied, it wets the hydrophilic binding pattern. (c) With the evaporation of water, the beads are dragged towards the center of the droplet. (d) The structure formed by the beads aggregating in the center of the binding pattern.

With constant bead concentration $C$, we can estimate the number $N$ of beads deposited on the hydrophilic areas as:

$N=C \cdot V / V_{b}$

where $V$ and $V_{b}=\pi d^{3} / 6$ are the respective volumes of the colloid droplet and the bead with diameter $d$. More accurate calculations of $N$ will take into account the droplet shape on the hydrophilic template. In this paper, we discuss models for two basic template patterns: rectangles and circular shapes.

\section{Rectangular pattern model}

Suppose the hydrophilic rectangular pattern is $L \times W$, with $L>>W$. The number of beads per unit length can be calculated by taking a segment of the colloid droplet with the length of $d$, which is the diameter of the beads (Fig. 2a); the area of the colloid droplet cross-section is $A$ and the volume of the droplet segment is $V$, which can be derived as: 
$A=W^{2}\left(\theta / \sin ^{2} \theta-\cot \theta\right) / 4$

$V=A \cdot d$

where $\theta$ is the contact angle of the colloid on the hydrophilic area.

Beads of diameter $d$ cannot occupy regions of the volume that have a minimum dimension smaller than $d$. Therefore, determining the number of beads simply by applying Eqs. 2 would create a significant error. The effective volume $V_{\text {eff }}$ of the droplet that can include beads is in general smaller than $V$. As shown in Fig. 2b, the effective volume $V_{\text {eff }}$ which the beads can occupy is $V_{\text {eff }}=A_{\text {eff }} d$, where the effective crosssection area is $A_{\text {eff }}$.

$A_{\text {eff }}=W_{1} \times d+W_{1}^{2}\left(\theta_{1} / \sin ^{2} \theta_{1}-\cot \theta_{1}\right) / 4$

where

$$
\begin{aligned}
& W_{l}=2 \sqrt{\left(\frac{W}{2 \sin \theta}-\frac{W}{2} \cot \theta-d\right)\left(\frac{W}{2 \sin \theta}+\frac{W}{2} \cot \theta+d\right)} \\
& \theta_{1}=\arcsin \left(\frac{W_{1} \sin \theta}{W}\right) .
\end{aligned}
$$

(a)

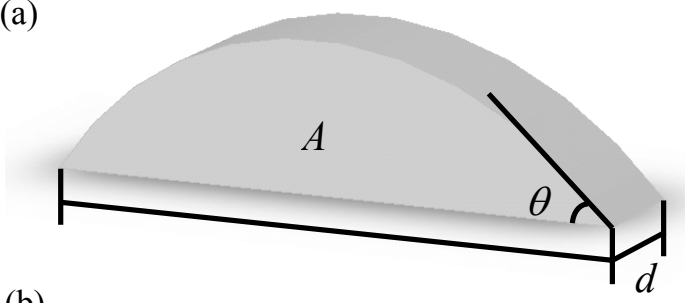

(b)

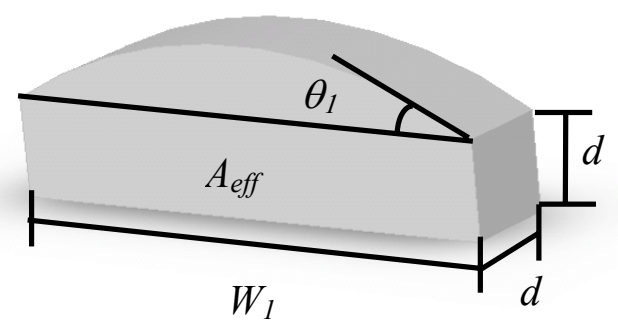

Figure 2. (a) Three-dimensional model of the colloid droplet segment formed on a rectangular pattern. (b) The effective volume of the droplet. $A_{\text {eff }}$ is the effective cross section area.

With the same rectangular binding sites, the effective area $A_{\text {eff }}$ varies with respect to the bead size $d$, which is illustrated in Table 1.

The number of beads per length $d$ can be estimated as:

$N=C \cdot V_{e f f} / V_{b}=C \cdot A_{e f f} d / V_{b}=6 C \cdot A_{e f f} / \pi d^{2}$
Table 1. Effective area $A_{\text {eff }}$ ratio changes with respect to the bead size $d$. We assume the contact angle $\theta$ to be $20^{\circ}$.

\begin{tabular}{||c|c||}
\hline \hline Bead size ratio $(d / W)$ & Effective area ratio $\left(A_{\text {eff }} / A\right)$ \\
\hline 0.0100 & 0.9951 \\
\hline 0.0200 & 0.9797 \\
\hline 0.0300 & 0.9517 \\
\hline 0.0400 & 0.9089 \\
\hline 0.0500 & 0.8474 \\
\hline 0.0600 & 0.7611 \\
\hline 0.0700 & 0.6380 \\
\hline 0.0800 & 0.4458 \\
\hline
\end{tabular}

\section{Circular pattern model}

Fig. 3 shows the three-dimensional model of a colloid droplet on a circular hydrophilic pattern. Similar to the discussion of the rectangular pattern above, we determine the number of beads deposited on a circular binding site with diameter $a$ as:

$N=C \cdot a^{3}\left((1 / \sin \theta-\cot \theta)\left(3+(1 / \sin \theta-\cot \theta)^{2}\right) / 8 d^{3}\right.$

From Eqs. 3-5, we can calculate the number of aggregated beads on patterns of various sizes. Conversely, the control of the nano-bead aggregate dimensions can be achieved by specific design of the hydrophilic patterns with respect to the bead size and bead concentration.

\section{EXPERIMENTAL SETUP}

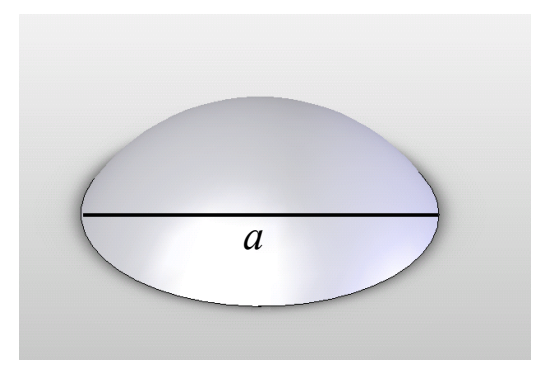

Figure 3. A three-dimensional model of the colloid droplet on a circular pattern with diameter $a$.

The substrate is prepared with a thermal oxidation layer of $400 \mathrm{~nm}$ on a silicon wafer. Then a thin layer of $\mathrm{TiW} / \mathrm{Au}$ $(30 \mathrm{~nm})$ is sputtered and patterned by photolithography and $\mathrm{Au} / \mathrm{TiW}$ etching for $20 \mathrm{~s}$ to expose the hydrophilic silicon oxide patterns. It is cleaned by two minutes of oxygen plasma, followed by soaking in $1 \mathrm{mM}$ ethanolic octadecanethiol solution to make the $\mathrm{Au}$ background hydrophobic. Colloid is then applied to cover the entire substrate, before the substrate touches the air-water interface, which removes the excessive colloid from the surface. In this step, the colloid is cleared from the hydrophobic area. Afterwards only the hydrophilic binding sites remain wetted. After the water evaporates in air, nano-beads are patterned on the binding sites. We use polystyrene nano-beads with the size ranging from $100 \mathrm{~nm}$ to $1 \mu \mathrm{m}$. 

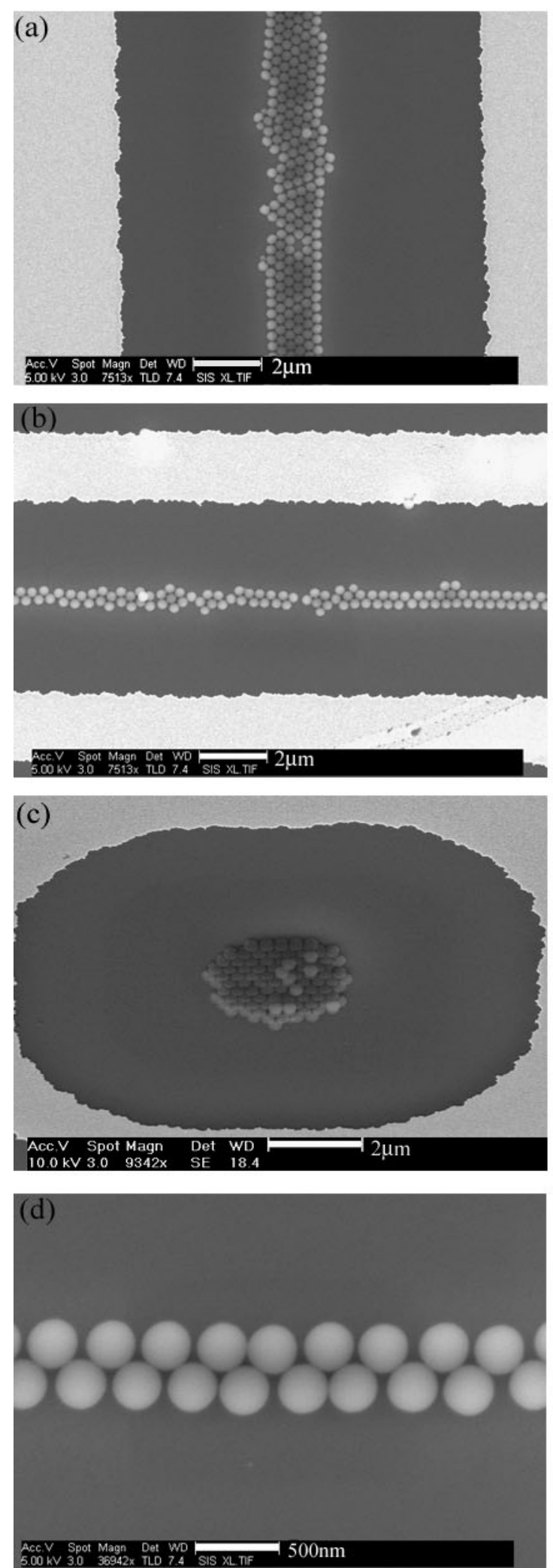

Figure 4. SEM images of nano-structures formed with $290 \mathrm{~nm}$ beads on different patterns. Arrays of beads on a rectangular shape with (a) $W=10 \mu \mathrm{m}$ and (b) $W=6 \mu \mathrm{m}$. (c) Beads arrange concentric to the circular binding site with diameter of $7 \mu \mathrm{m}$. (d) A zoomed-in image of a double-strand bead array.

\section{Results}

Experimental results show agreement with our models. With the same bead size and concentration, different threedimensional structures are observed. Fig. 4 shows results of various nano-structures formed from colloid with $10 \mathrm{wt} . \%$ $290 \mathrm{~nm}$ beads on different binding shapes and sizes. For comparison, we calculated the number of beads based on the models discussed in the previous section, with measured contact angle $\theta$ of water on silicon dioxide at approximately $20^{\circ}$. Table 2 shows both the calculated and experimental values.

Table 2. Comparisons between calculated and experimental data of nano-structure sizes formed from colloid with 10 wt.\% 290nm beads.

\begin{tabular}{||l|l|l|l|}
\hline $\begin{array}{c}\text { Binding site } \\
\text { shape and size }\end{array}$ & $\begin{array}{l}\text { Rectangle } \\
W=10 \mu \mathrm{m}, \\
L>>W\end{array}$ & $\begin{array}{l}\text { Rectangle } \\
W=6 \mu \mathrm{m}, \\
L>>W\end{array}$ & $\begin{array}{l}\text { Circle } \\
a=7 \mu \mathrm{m}\end{array}$ \\
\hline Formula & Eq. 4 & Eq. 4 & Eq. 5 \\
\hline $\begin{array}{l}\text { Cal. } N \\
\text { (\# of beads) }\end{array}$ & 7 & 2 & 94 \\
\hline $\begin{array}{l}\text { Exp. } N \\
\text { (\# of beads) }\end{array}$ & $5 \sim 6$ & $2 \sim 3$ & 101 \\
\hline
\end{tabular}

Figs. 5a-c show the different nano-structures formed on identical patterns with $100 \mathrm{~nm}, 290 \mathrm{~nm}$ and $1 \mu \mathrm{m}$ beads. For similar binding site dimensions, different bead sizes and concentrations can result in drastic changes in the nanostructures: a discontinuous $1 \mu \mathrm{m}$ single bead line in Fig. 6a and multiple layered $100 \mathrm{~nm}$ nano-structures in Fig. 6b.

\section{Conclusions AND Discussions}

In conclusion, we have investigated an approach to construct nano-structures by colloidal aggregation, with accordant simulation and experimental results. The advantages of this technique include: (1) simple surface patterning techniques suffice to construct the binding templates for nano-scale structures; (2) no specific surface treatment is required for the beads; (3) with the proposed models, we can predict and control the shape of the three-dimensional structures.

This technique can be applied as an enabling technology for building nano-systems, e.g. nano-bead based biochemical sensing elements. More generally, it can be used as a nanofabrication technique for transforming micro patterns into nano-structures at one to two size scales below the optical lithography limit. This could lead to new, low-cost methods in the manufacture of nanoscale masks and circuits. 

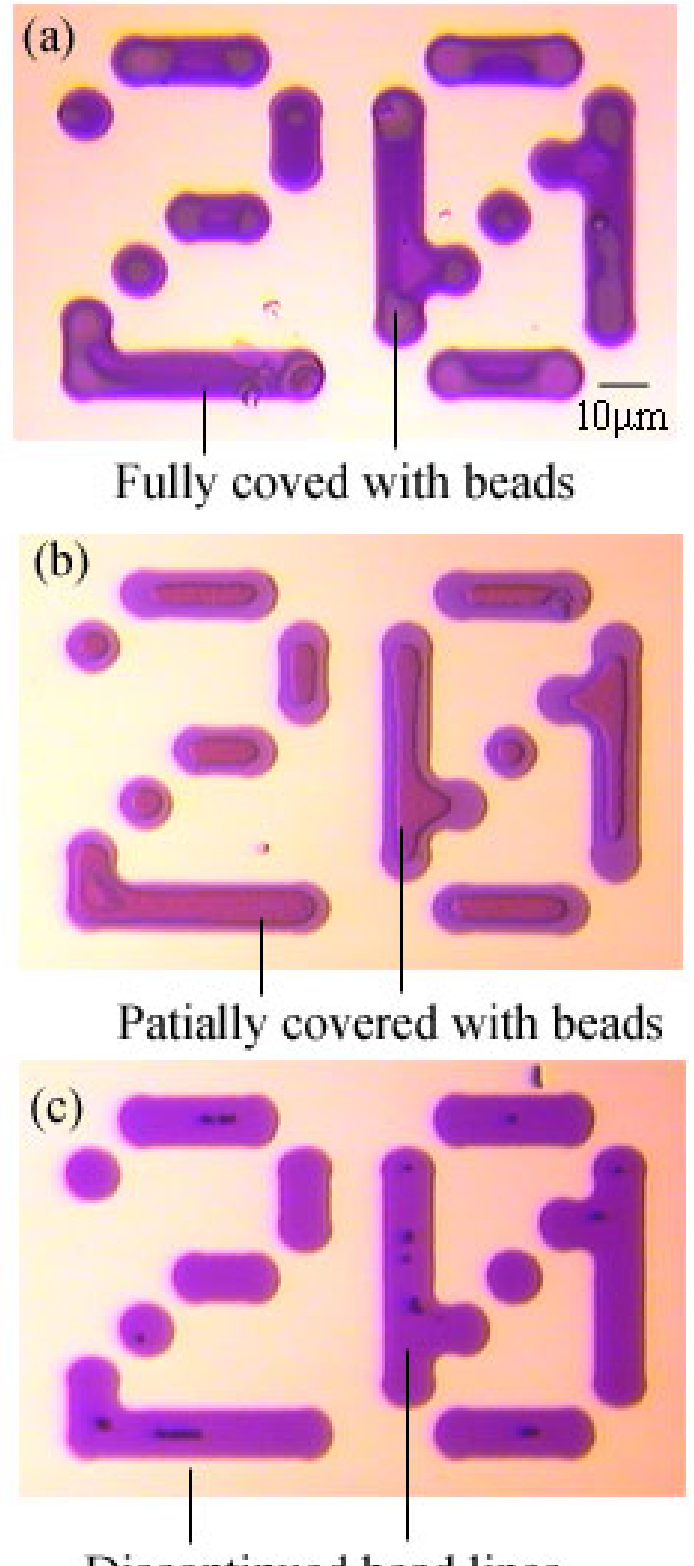

\section{Discontinued bead lines}

Figure 5. Different nano-structures formed on identical patterns by beads of different sizes: (a) 100nm (b) $290 \mathrm{~nm}$ (c) $1 \mu \mathrm{m}$ in diameter. Note: the pattern in (a) is packed with multiple layers of beads, showing as different shadows.

\section{REFERENCES}

[1] H. McNally, M. Pingle, S.W. Lee, D. Guo, D.E. Bergstrom, R. Bashir, "Self-assembly of micro- and nanoscale particles." Applied Surface Science 214 (2003) 109119.

[2] H. Andersson, C. Jönsson, C. Moberg and G. Stemme, "Self-assembled and self-sorted array of chemically active beads for analytical and biochemical screening." Talanta 56 (2002) 301-308.

[3] S.W. Lee, H.A. McNally, D. Guo, M. Pingle, D.E. Bergstrom, R. Bashir, "Electric-field-mediated assembly of
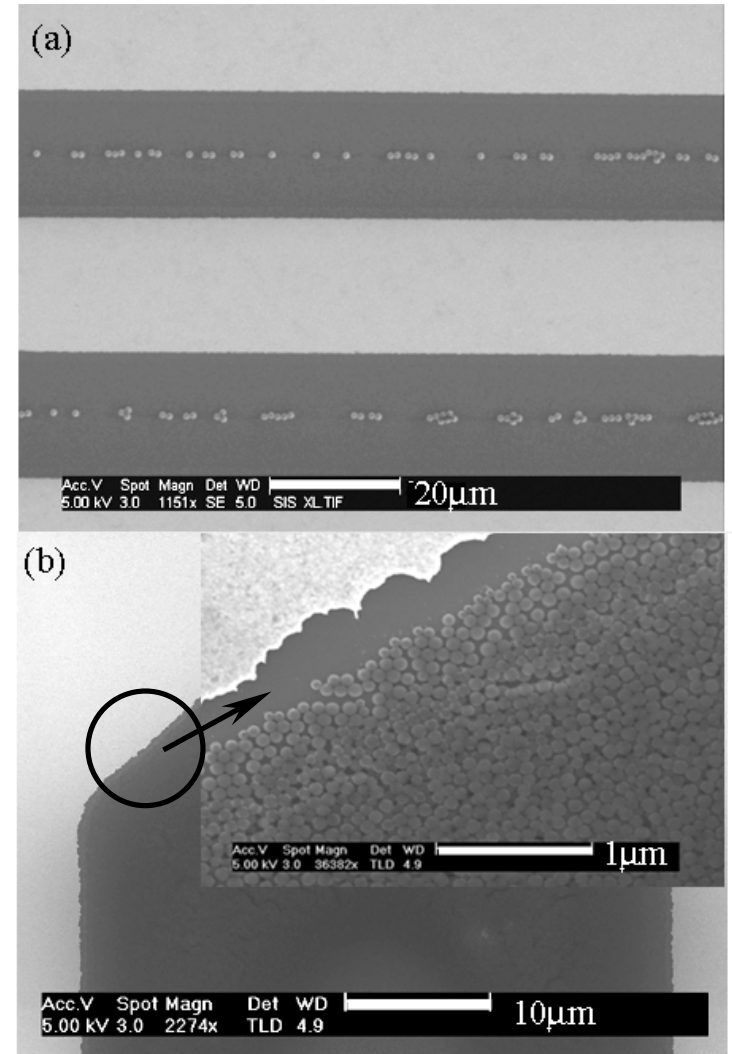

Figure 6. Binding sites with similar size result in different nano-structures formed by different beads and different concentrations: (a) $1 \mathrm{wt}$ \% concentration $1 \mu \mathrm{m}$ beads form single sparse line, (b) 10 wt.\% concentration $100 \mathrm{~nm}$ beads form multi-layer nano-structure.

silicon islands coated with charged molecules." Langmuir 18 (8) (2002) 3383-3386.

[4] Z. Zhong, B. Gates, Y. Xia, "Soft lithographic approach to the fabrication of highly ordered $2 \mathrm{D}$ arrays of magnetic nanoparticles on the surfaces of silicon substrates." Langmuir 16 (2000) 10369-10375.

[5] U. Srinivasan, R. Howe, D. Liepmann, "Microstructure to substrate self-assembly using capillary forces." ASME/IEEE Journal of Microelectromechanical Systems 10 (2001) 1724.

[6] A. Terfort, N. Bowden, G. M. Whitesides, "Threedimensional self-assembly of millimetre-scale components." Nature 386 (1997).

[7] X. Xiong, Y. Hanein, J. Fang, Y. Wang, W. Wang, D. T. Schwartz, K. F. Böhringer, "Controlled Multi-Batch SelfAssembly of Micro Devices." ASME/IEEE Journal of Microelectromechanical Systems 12 (2) (2003) 117-127.

[8] P.A. Kralchevsky, N.D. Denkov, "Capillary forces and structuring in layers of colloid particles." Current Opin. Col. \& Int. Sci. 6 (2001) 383-401.

[9] K. Nagayama, "Two-dimensional self-assembly of colloids in thin liquid films, "Colloids Surfaces A: Physicochem. Eng. Aspects 109 (1996) 363-374 